\section{Budesonid nur einmal wöchentlich?}

\author{
Liposomen werden seit einiger Zeit als Trägersysteme für Arznei- \\ mittel verwendet. Sie verbessern die Verträglichkeit und führen \\ durch die protrahierte Pharmakafreisetzung zu einem Depoteffekt. \\ Ob ein solcher Effekt mit Hilfe von neuartigen, Budesonid tragenden \\ Liposomen auch in der Lunge zu erzielen ist, wurde jetzt an Mäusen \\ untersucht.
}

$\mathrm{H}$ erkömmliche Liposomen haben den Nachteil, relativ rasch von den Zellen des retikuloendothelialen Systems erkannt und eliminiert zu werden. Darüber hinaus sind sie speziell in der Asthmatherapie als Depotträger wenig geeignet, weil die Glukokortikoide zu schnell aus ihnen heraus diffundieren. Dies will man durch den Einsatz von neuartigen, sterisch stabilisierten Liposomen vermeiden.

In einer experimentellen Studie wurden Mäuse mit Ovalbumin sensibilisiert. Dadurch entwickeln die Tiere Entzündungszeichen, die das menschliche Asthma relativ gut simulieren. Die Asthmamäuse erhielten dann in verne- belter Form über 4 Wochen einmal wöchentlich $20 \mu \mathrm{g}$ Budesonid, eingekapselt in sterisch stabilisierte Liposomen, $20 \mu \mathrm{g}$ nicht eingekapseltes Budesonid

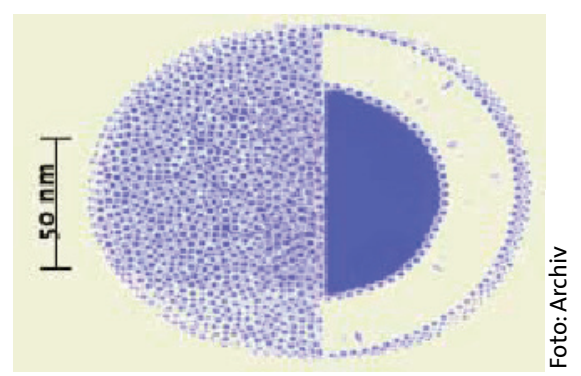

Eingehüllt in ihre Phospholipid-Doppelmembran transportieren Liposomen Wirkstoffe an ihren Einsatzort. einmal täglich, $20 \mu \mathrm{g}$ Budesonid einmal wöchentlich in herkömmlichen Liposomen oder einmal wöchentlich die sterisch stabilisierten Liposomen ohne Wirkstoff.

Die wöchentliche Inhalation des in sterisch stabilisierte Liposomen eingebetteten Budesonids war genauso effektiv wie die tägliche Inhalation von $\mathrm{Bu}$ desonid, nachgewiesen anhand der Entzündungsreaktion an der Lunge, der peripheren Bluteosinophilen, der Peroxidaseaktivität der Eosinophilen und der Gesamt-IgE-Spiegel. In den beiden anderen Gruppen blieben diese Parameter unverändert und unterschieden sich nicht von denen von Kontrollmäusen.

Fazit: Die wöchentliche inhalative Gabe von Budesonid, eingekapselt in sterisch stabilisierte Liposomen, könnte eine neue Therapieoption für Asthmapatienten werden.

Konduri KS et al. Efficacy of liposoma budesonide in experimental asthma. J Allergy Clin Immunol 2003; 111: 321-7

\title{
Inhalative Gukokortikoide - Dosisreduktion fast immer möglich
}

\begin{abstract}
Allgemein gelten inhalative Glukokortikoide als gut verträglich. Trotzdem empfehlen Richtlinien zur Asthmatherapie den Versuch einer Dosisreduktion, sobald der Patient gut eingestellt ist (Stepdown). Diese Empfehlung basiert überwiegend auf Erfahrungen von Experten. Eine kontrollierte Studie liefert jetzt eine Bestätigung.
\end{abstract}

Fom orscher der Universität Glasgow untersuchten im Rahmen einer multizentrischen, randomisierten, doppelblinden Parallelgruppenstudie 259 erwachsene Asthmatiker mit einem mindestens seit 12 Monaten bestehenden Asthma. Die Patienten waren auf mindestens $800 \mu \mathrm{g} / \mathrm{d}$ inhalatives Beclometasondipropionat oder entsprechende Äquivalente von Budesonid bzw. Fluticasonpropionat eingestellt, die mittlere Dosis betrug $1.430 \mu \mathrm{g} / \mathrm{d} \mathrm{Be}-$ clometasondipropionat. Sie erhielten über ein Jahr entweder ihre gewohnte Dosis weiter oder eine um 50\% redu- zierte Dosis, sobald das Asthma stabil war. Im Rahmen der regelmäßigen Untersuchungen wurden jeweils neue Inhalationsbehälter ausgegeben. Diese waren für beide Gruppen äußerlich identisch und lediglich mit der Aufschrift „übliche Dosis“ oder „reduzierte Dosis" versehen, wobei aber nur die Patienten der Stepdown-Gruppe tatsächlich die reduzierte Dosis applizierten.

Im Beobachtungszeitraum traten in beiden Gruppen Asthmaexazerbationen mit statistisch vergleichbarer Häufigkeit auf (Stepdown-Gruppe 31\%, Kontrollgruppe 26\%; p = 0,354). Auch die Anzahl der Arztkontakte oder Parameter des allgemeinen Gesundheitsstatus unterschieden sich nicht signifikant. Das galt auch für den Verbrauch an oralen Glukokortikoiden, die zur Therapie der Exazerbationen zusätzlich verordnet wurden. Insgesamt war bei 83\% der Patienten in der StepdownGruppe eine Rücktitration möglich. Im Mittel reduzierte sich die Dosis um $348 \mu \mathrm{g} / \mathrm{d}$, was einem Unterschied von $25 \%$ im Vergleich zur Kontrollgruppe entspricht.

Fazit: Bei Asthmatikern mit guter Symptomenkontrolle und ohnehin relativ hoher täglicher Glukokortikoiddosis ist es möglich, diese Dosis zu reduzieren, ohne zusätzliche Exazerbationen befürchten zu müssen. Dies gilt auch für Raucher, die in dieser Studie ausdrücklich zugelassen waren. $\quad b k$

Hawkins $\mathbf{G}$ et al. Stepping down inhaled corticosteroids in asthma: randomised controlled trial. BMJ 2003; 326: 1115-8 